\title{
miR-15a-3p affects the proliferation, migration and apoptosis of lens epithelial cells
}

\author{
SHU-JUN LIU ${ }^{1 *}$, WEN-TING WANG ${ }^{2 *}$, FENG-LAN ZHANG $^{1}$, YONG-HONG YU ${ }^{1}$, \\ HUA-JUN YU ${ }^{1}$, YAN LIANG ${ }^{1}$, NING LI ${ }^{2}$ and YUAN-BIN LI ${ }^{1}$ \\ ${ }^{1}$ Department of Ophthalmology, ${ }^{2}$ Central Laboratory, The Affiliated Yantai Yuhuangding Hospital of Qingdao University, \\ Yantai, Shandong 264000, P.R. China
}

Received March 26, 2018; Accepted October 29, 2018

DOI: $10.3892 / \mathrm{mmr} .2018 .9738$

\begin{abstract}
The present study investigated the effect of microRNA (miR)-15a-3p on the proliferation, migration and apoptosis of lens epithelial cells and its potential mechanism, in order to further elucidate the pathogenesis of age-related cataracts (ARCs). The HLE-B3 human lens epithelial cell line was transfected with miR-15a-3p mimic. Expression of the miR-15a-3p mimic was measured by fluorescence-based reverse transcription-quantitative polymerase chain reaction analysis. Cell proliferation, apoptosis, invasion and migration were investigated using MTT and plate clone formation assays, terminal deoxynucleotidyl transferase dUTP nick end labeling and flow cytometry, and a wound healing assay and Transwell assay, respectively. The protein expression levels of B-cell lymphoma 2 (BCL2) and myeloid cell leukemia sequence 1 (MCL1) were also compared between transfected and wild-type HLE-B3 cells by western blot analysis. The results showed that transfection with the miR-15a-3p mimic significantly suppressed the proliferation of HLE-B3 cells, induced cell apoptosis and increased the proportion of early apoptotic cells. The migration of HLE-B3 cells was
\end{abstract}

Correspondence to: Dr Yuan-Bin Li, Department of Ophthalmology, The Affiliated Yantai Yuhuangding Hospital of Qingdao University, 20 Yuhuangding East Road, Yantai, Shandong 264000, P.R. China E-mail: ytliyuanbin@163.com

Ms. Ning Li, Central Laboratory, The Affiliated Yantai Yuhuangding Hospital of Qingdao University, 20 Yuhuangding East Road, Yantai, Shandong 264000, P.R. China

E-mail: ningning305@126.com

*Contributed equally

Abbreviations: ARC, age-related cataract; RT-qPCR, reverse transcription-quantitative polymerase chain reaction; RNU6B, RNA U6 small nuclear 2; OD, optical density; TUNEL, terminal deoxynucleotidyl transferase dUTP nick end labeling; PI, propidium iodide; DAPI, 4',6-diamidino-2-phenylindole; SD, standard deviation

Key words: microRNA-15a, human lens epithelial cell, age-related cataract significantly inhibited following transfection with miR-15a-3p mimic $(P<0.01)$, whereas cell invasion was unaffected $(P>0.05)$. In addition, reduced protein levels of BCL2 and MCL1 were observed in the miR-15a-3p mimic-transfected HLE-B3 cells $(\mathrm{P}<0.01)$. In conclusion, miR-15a-3p may suppress cell proliferation and migration, and induce cell apoptosis in lens epithelial cells through inhibiting the expression of BCL2 and MCL1, which contributes to the onset of ARCs.

\section{Introduction}

Age-related cataract (ARC) is one of the major causes of visual impairment or visual loss. Despite various in-depth investigations on ARC in previous years, surgical replacement with an artificial lens remains the only cure for ARC due to its complicated pathogenesis and unclear mechanism. Therefore, it is of importance to further examine the pathogenic mechanism of ARC, and to develop novel therapies for its prevention and treatment.

Lens epithelial cells can differentiate into lens fibers, which are important in the biological function of the lens. Previous studies have found that, during the development of ARC, several genes undergo structural or functional alterations (1). This leads to the apoptosis of lens epithelial cells and degeneration of lens proteins and ultimately causes all types of cataract, with the exception of congenital cataracts (2). MicroRNAs (miRNAs) are a small type of RNA molecule that are extensively present in animals and plants and are involved in post-transcriptional gene regulation. They are also involved in a variety of other biological activities, including cell proliferation, differentiation and apoptosis (3). Previous studies have identified miRNA (miR)-15a as an active contributor in the apoptosis of several cell types $(4,5)$. miR-15a is the natural antisense interfering RNA of the proto-oncogene B-cell lymphoma 2 (BCL2). The nine bases at the 5 'end of miR-15a are complementary to bases 3,287-3,279 of BCL2 (4). Their combination results in downregulation of the expression of BCL2 and thus promotes cell apoptosis. As another member of the BCL2 family, the expression of myeloid cell leukemia sequence 1 (MCL1) can also be inhibited by miR-15a, which promotes apoptosis (6). Our previous study identified elevated expression of miR-15a-3p in the lens epithelial cells of patients with ARC (7). Is it possible that the interaction between 
miR-15a-3p and anti-apoptotic proteins BCL2 or MCL1 leads to the apoptosis of lens epithelial cells and altered proliferation and differentiation of lens epithelial cells, ultimately causing ARC. To confirm this hypothesis, the present study transformed miR-15a-3p mimic into the HLE-B3 human lens epithelial cell line and examined its proliferation, migration and apoptosis. The study also aimed to reveal the role of miR-15a-3p in the pathogenesis of ARC.

\section{Materials and methods}

Ethics. The present study was approved by the Institutional Review Board of Yantai Yuhuangding Hospital (Yantai, China).

Cell culture and transfection. The HLE-B3 cells were obtained from Shanghai Genechem Co., Ltd. (Shanghai, China) and were maintained in DMEM (Gibco; Thermo Fisher Scientific, Inc., Waltham, MA, USA) supplemented with $10 \%$ FBS (Hyclone; GE Healthcare Life Sciences, Logan, UT, USA), under a $37^{\circ} \mathrm{C}$ humidified, $5 \% \mathrm{CO}_{2}$ environment. The cells were transfected with miR-15a-3p mimic, miR-15a-3p mock or mimic negative control (NC) from GenePharma (Shanghai, China) using Lipofectamine RNAiMax (Invitrogen; Thermo Fisher Scientific, Inc.), following the manufacturer's protocol. After $48 \mathrm{~h}$, the cells were harvested for subsequent experiments.

Reverse transcription-quantitative polymerase chain reaction (RT-qPCR) analysis. The miRNA expression levels were determined by RT-qPCR analysis. In brief, total RNA was extracted from the cultured cells using an RNA extraction kit (Thermo Fisher Scientific, Inc.) according to the manufacturer's protocol. Then, $2 \mu \mathrm{g}$ total RNA was reverse transcribed using ReverTra Ace (Toyobo Life Science, Osaka, Japan). RT-qPCR analysis for miR-15a-3p was conducted using the ABI7500 (Applied Biosystems; Thermo Fisher Scientific, Inc.) in accordance with the manufacturer's protocol. The PCR amplifications were performed using a $50 \mu \mathrm{l}$ final reaction mixture containing $25 \mu \mathrm{l}$ SYBR Green PCR Master mix (Platinum SYBR Green qPCR SuperMix-UDG kit, Life Technologies; Thermo Fisher Scientific, Inc.), $1.5 \mu \mathrm{l}$ primer $\operatorname{mix}(10 \mu \mathrm{M})$ and $1 \mu \mathrm{l}$ template. Cycling conditions were: $50^{\circ} \mathrm{C}$ for $2 \mathrm{~min} ; 95^{\circ} \mathrm{C}$ for $5 \mathrm{~min}$; followed by 40 cycles of $95^{\circ} \mathrm{C}, 10 \mathrm{sec}$ and $60^{\circ} \mathrm{C}, 45 \mathrm{sec}$. The melting curve analysis and agarose gel electrophoresis were used to confirm the specific PCR products. The expression of RNA U6 small nuclear 2 (RNU6B) was also measured as an internal control. The primer sequences were as follows: miR-15a-3p RT, 5'-CTCAACTGGTGTCGTGGAGTCGGCA ATTCAGTTGAGTGAGGCA-3'; miR-15a-3p, forward 5'-ACA CTCCAGCTGGGCAGGCCATATTGTGCTGCCTC-3' and reverse 5'-TGGTGTCGTGGAGTCG-3'; RNU6B, forward 5'-CTCGCTTCGGCAGCACA-3' and reverse 5'-AACGCT TCACGAATTTGCGT-3'. Product specificity was confirmed by melting curve analysis. All experiments were performed in triplicate with three separate samples. All results were determined as the expression of $\mathrm{miR}-15 \mathrm{a}-3 \mathrm{p}$ relative to that of the internal control in cell lines using the $2^{-\Delta \Delta \mathrm{Cq}}$ method (8).

Cell proliferation assay. MTT assays were performed to assess the proliferative ability of the transfected HLE-B3 cells.
In each well of a 96 -well plate, $3 \times 10^{3}$ cells were seeded and, $24 \mathrm{~h}$ later, were transfected with 5 pmol miR-15a-3p mimic, miR-15a-3p mock or mimic NC (GenePharma). At 0, 24, 48, 72 and $96 \mathrm{~h}$ following transfection, $20 \mu \mathrm{l}$ of MTT $(5 \mathrm{mg} / \mathrm{ml}$, Sigma-Aldrich; Merck KGaA, Darmstadt, Germany) was added to the wells and incubated at $37^{\circ} \mathrm{C}$ for $2 \mathrm{~h}$. Subsequently, the culture medium was replaced with $150 \mu \mathrm{l}$ of dimethylsulfoxide (Sigma-Aldrich; Merck KGaA) and then agitated at $0.04 \mathrm{x} \mathrm{g}$ for $15 \mathrm{~min}$ at room temperature. The optical density (OD) value of each well was then evaluated using the ELISA microplate reader (Bio-Rad Laboratories, Inc., Hercules, CA, USA) at a wavelength of $490 \mathrm{~nm}$. The experiments were performed in triplicate and repeated at least three times.

Plate colony formation experiments. Cells $\left(1 \times 10^{4}\right)$ in logarithmic growth phase were seeded onto 6-cm plates and cultured for 2-3 weeks. When visible colonies had appeared, the cells were washed with PBS twice, fixed in methanol for $15 \mathrm{~min}$, stained with crystal violet for $20 \mathrm{~min}$, washed with water, and air dried. The number of visible colonies was counted under an inverted microscope (IX51; Olympus Corporation, Tokyo, Japan) using a transparent film with grids. Colony formation rate $=$ (number of colonies/number of inoculated cells) x $100 \%$.

Cell apoptotic rate assay. Annexin V-FITC/propidium iodide (PI) staining was conducted to assess the apoptotic rate of cells using flow cytometry (BD Biosciences, Franklin Lakes, NJ, USA). Briefly, following transfection, the HLE-B3 cells $\left(1 \times 10^{6}\right)$ were collected and stained with Annexin V-FITC/PI using an apoptosis detection kit (BD Biosciences). Following $15 \mathrm{~min}$ of incubation at room temperature in the dark, the apoptotic cell percentages were measured through flow cytometry. The Annexin V immunofluorescence was presented on the $\mathrm{x}$-axis and the plasma membrane integrity was presented on the $\mathrm{y}$-axis. Each experiment was conducted in triplicate.

Wound-healing assay. The cells were inoculated in six-well plates and routinely cultured until they reached $70-80 \%$ confluence. Cross lines were introduced using a $200-\mu 1$ sterile pipette tip. The cells were washed three times with sterile PBS to remove the cells from the scratched region and were then continually cultured in serum-free culture medium. Images of the cells were captured at 0 and $24 \mathrm{~h}$ post-wounding. Cell migration distance $=$ distance at $0 \mathrm{~h}$-distance at $24 \mathrm{~h}$.

Transwell assay. Transwell chamber inserts (BD Biosciences) with (for invasion) or without (for migration) Matrigel (BD Biosciences) were used. The cells were transfected with miR-15a-3p mimics, inhibitors, mimic NC or inhibitor NC. At $6 \mathrm{~h}$ following transfection, $1 \times 10^{4}$ transfected cells in $200 \mu \mathrm{l}$ serum-free DMEM were seeded in the upper chamber of the insert. The bottoms of the inserts were incubated in medium with $10 \%$ FBS. Following $36 \mathrm{~h}$ (for migration) or $48 \mathrm{~h}$ (for invasion), cells on the upper membrane were removed using a cotton swab. Those cells that had migrated or invaded to the bottom of the inserts were stained with crystal violet $(1 \mathrm{mg} / \mathrm{ml}$, Sigma-Aldrich; Merck KGaA) for $15 \mathrm{~min}$ at room temperature and counted using a Leica DMIRB inverted microscope (x200 magnification). The experiments were performed in triplicate and repeated at least three times. 
Western blot analysis. Western blot analysis was performed as previously described (9). Total proteins were extracted in a lysis buffer $(20 \mathrm{mM}$ Tris- $\mathrm{HCl}, \mathrm{pH} 7.5,150 \mathrm{mM} \mathrm{NaCl}, 5 \mathrm{mM}$ EDTA, pH 8.0, $5 \mathrm{mM} \mathrm{NaPPi}, 1 \mathrm{mM}$ sodium orthovanadate $\left(\mathrm{Na}_{3} \mathrm{VO}_{4}\right), 1 \mathrm{mM}$ PMSF, $1 \% \mathrm{NP}-40$, and $10 \mu \mathrm{g} / \mathrm{ml}$ each aprotinin and leupeptin). Tissue lysates were centrifuged at $10,000 \mathrm{~g}$ for $10 \mathrm{~min}$ at $4^{\circ} \mathrm{C}$. Sample concentration was detected by BCA. Then $20 \mu \mathrm{g}$ protein was subjected to $10 \%$ SDS-PAGE, transferred onto PVDF membranes and followed by immunoblotting. Following blocking with TBS containing 5\% bovine serum albumin (BSA; Sigma-Aldrich; Merck KGaA) and $0.1 \%$ Tween-20, the membrane was incubated with appropriate primary antibodies at $4^{\circ} \mathrm{C}$ overnight, followed by incubation with a peroxidase-conjugated goat anti-mouse $\operatorname{IgG}(1: 5,000$; ZB-2305, ZSGB-BIO, Beijing, China) at room temperature for $1 \mathrm{~h}$. GAPDH was used as a loading control. The primary antibodies used were as follows: Anti-BCL2 (1:1,000; sc-509, Santa Cruz Biotechnology, Inc., Santa Cruz, CA, USA), anti-MCL1 (1:1,000; sc-53951, Santa Cruz Biotechnology) and anti-GAPDH (1:1,000; sc-293335, Santa Cruz Biotechnology, Inc.) For quantification, densitometric analysis was performed by analyzing the grey level intensity of target bands derived from scanned films, processed using ImageJ version 1.47 (National Institutes of Health, Bethesda, MD, USA).

Terminal deoxynucleotidyl transferase dUTP nick end labeling (TUNEL) assay. The cells (100 $\mu \mathrm{l}$ of $2 \times 10^{4}$ cells) were added into each well of a 96-well plate and incubated overnight, followed by cell transfection. Subsequently, the cells were cultured in conventional conditions for $48 \mathrm{~h}$. The medium was discarded and the cells were fixed with 4\% paraformaldehyde for 30 min prior to washing with PBS. Following the addition of $0.3 \% \mathrm{H}_{2} \mathrm{O}_{2}$-methanol solution, the cells were incubated at room temperature for another $30 \mathrm{~min}$. Following PBS washing, $0.1 \%$ Triton X-100 in $0.1 \%$ sodium citrate solution was added into wells for 2 min incubation in an ice-bath to permeabilize cells, followed by PBS washing. With the addition of $50 \mu \mathrm{l}$ TUNEL reagent (In Situ Cell Death Detection kit, Fluorescein; Roche Diagnostics, Indianapolis, IN, USA), the cells were cultured in a humidified incubator at $37^{\circ} \mathrm{C}$ for $60 \mathrm{~min}$. Following three PBS washes, $50 \mu 1$ of 4',6-diamidino-2-phenylindole was added to cells, followed by incubation at $37^{\circ} \mathrm{C}$ in the dark and another three PBS washes. The cells were examined under a fluorescence microscope and images were captured.

Statistical analysis. All statistical analyses were performed with SPSS for Windows version 18.0 (SPSS, Inc., Chicago, IL, USA). Data are presented as the mean \pm standard deviation (SD). Error bars represent the SD of three independent experiments. Differences between groups were compared using one-way analysis of variance with a post hoc test (LSD). All statistical analyses were two-sided, and $\mathrm{P}<0.05$ was considered to indicate a statistically significant difference.

\section{Results}

Expression of miR-15a-3p in transfected HLE-B3 cells causes morphological changes. The HLE-B3 cells were transfected with miR-15a-3p mimic (miR-15a), miR-15a-3p mock and
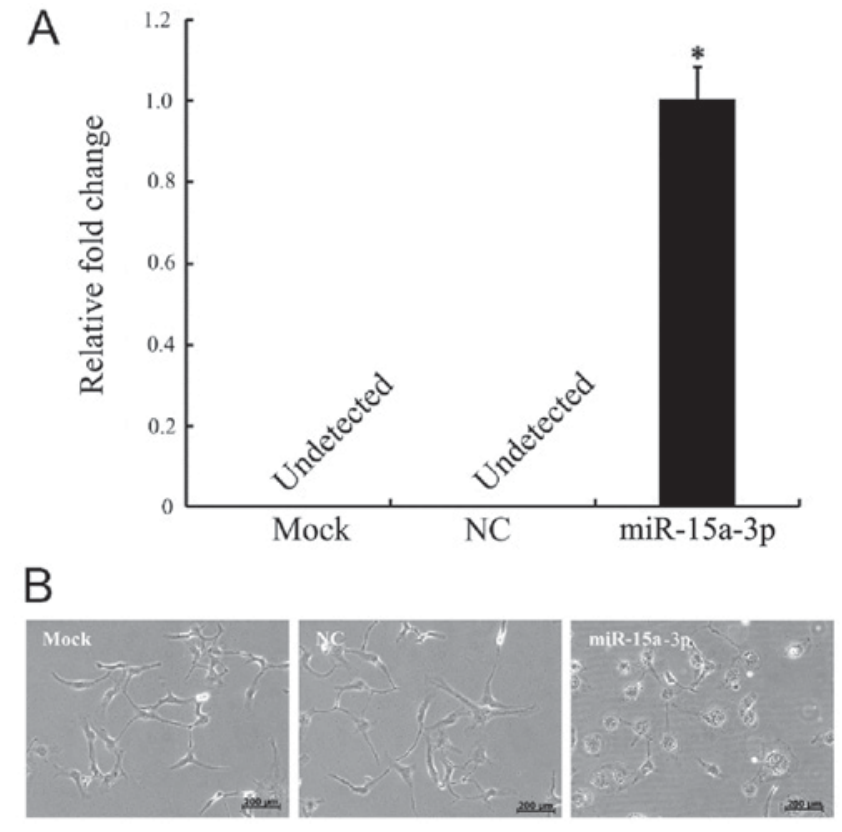

Figure 1. Expression of miR-15a-3p causes morphological changes in HLE-B3 cells. (A) Reverse transcription-quantitative polymerase chain reaction analysis demonstrating the expression of miR-15a in transfected HLE-B3 cells. (B) Comparison of the morphology of miRNA-15a-expressing HLE-B3 cells with the NC and mock groups of cells. " $\mathrm{P}<0.01$. miR, microRNA; NC, negative control.

mimic NC. No expression of miR-15a-3p was present in the NC cells, as detected by RT-qPCR analysis, whereas there was significantly elevated expression of miR-15a-3p in the miR-15a-3p mimic-transfected cells (Fig. 1A).

Morphological changes occurred in the miR-15a-3p cells. The normal HLE-B3 cells were irregular polygon-shaped with interactions that formed network structures. The morphology of the miR-15a-3p mimic-transfected cells began changing from $24 \mathrm{~h}$ post-transfection. The cells became large and round, with unclear boundaries and reduced networks (Fig. 1B).

Expression of miR-15a-3p inhibits HLE-B3 cell proliferation. The effect of the expression of miR-15a-3p on HLE-B3 cell proliferation was investigated using an MTT assay. From $48 \mathrm{~h}$ post-transfection, cell proliferation in the miR-15a group was increasingly inhibited. On days 3, 4 and 5 post-transfection, the proliferation rates of the miR-15a cells were significantly lower than those of the Mock and NC cells $(\mathrm{P}<0.01)$ (Fig. 2A).

The proliferation of transfected HLE-B3 cells was further confirmed using a plate colony formation assay. The results showed that, compared with the NC and mock cells, the colony-forming ability of the miR-15a cells was significantly attenuated (Fig. 2B and C).

Expression of miR-15a-3p induces HLE-B3 cell apoptosis. The in situ apoptosis of the transfected HLE-B3 cells was investigated using a TUNEL assay. The results showed that evident apoptotic signals were detected in the miR-15a cells compared with the mock and NC cells, suggesting that miR-15a-3p may induce HLE-B3 cell apoptosis (Fig. 3A). Following Annexin V-FITC/PI 

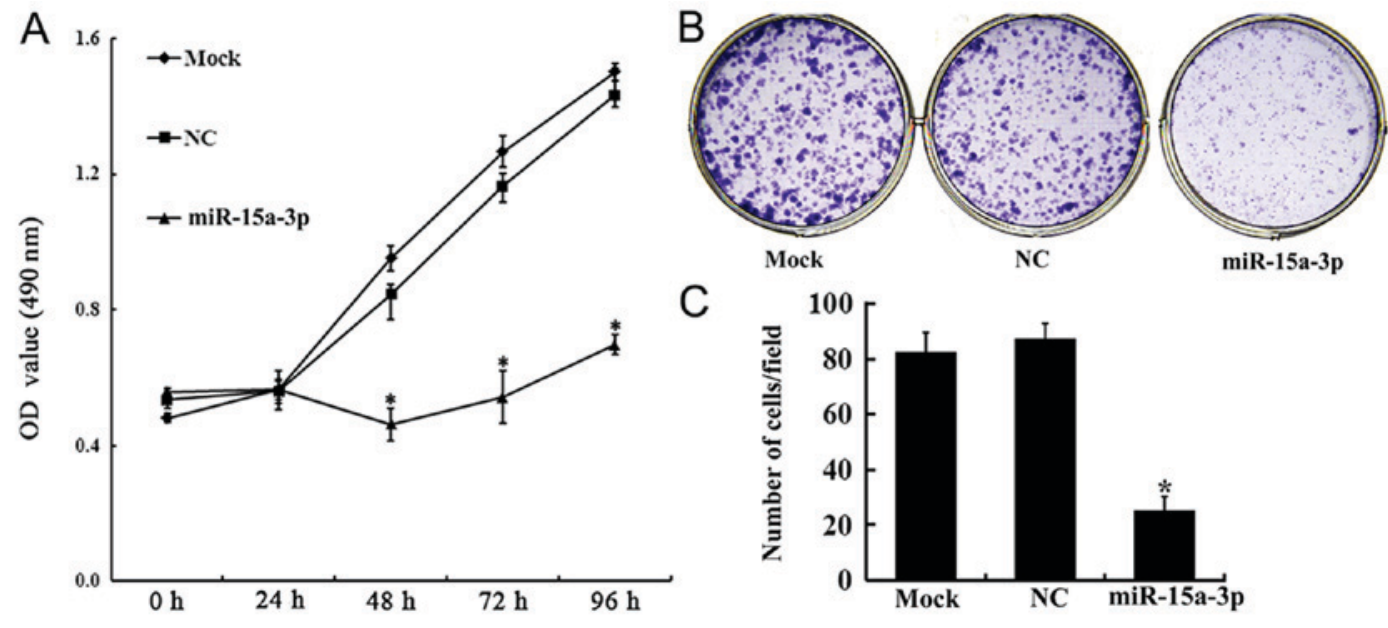

Figure 2. Expression of miR-15a-3p inhibits HLE-B3 cell proliferation. (A) MTT assay showing the proliferation of transfected HLE-B3 cells. (B) Colony formation of transfected HLE-B3 cells. (C) Cell count for the colony formation assay. "P<0.01. miR, microRNA; NC, negative control; OD, optical density.
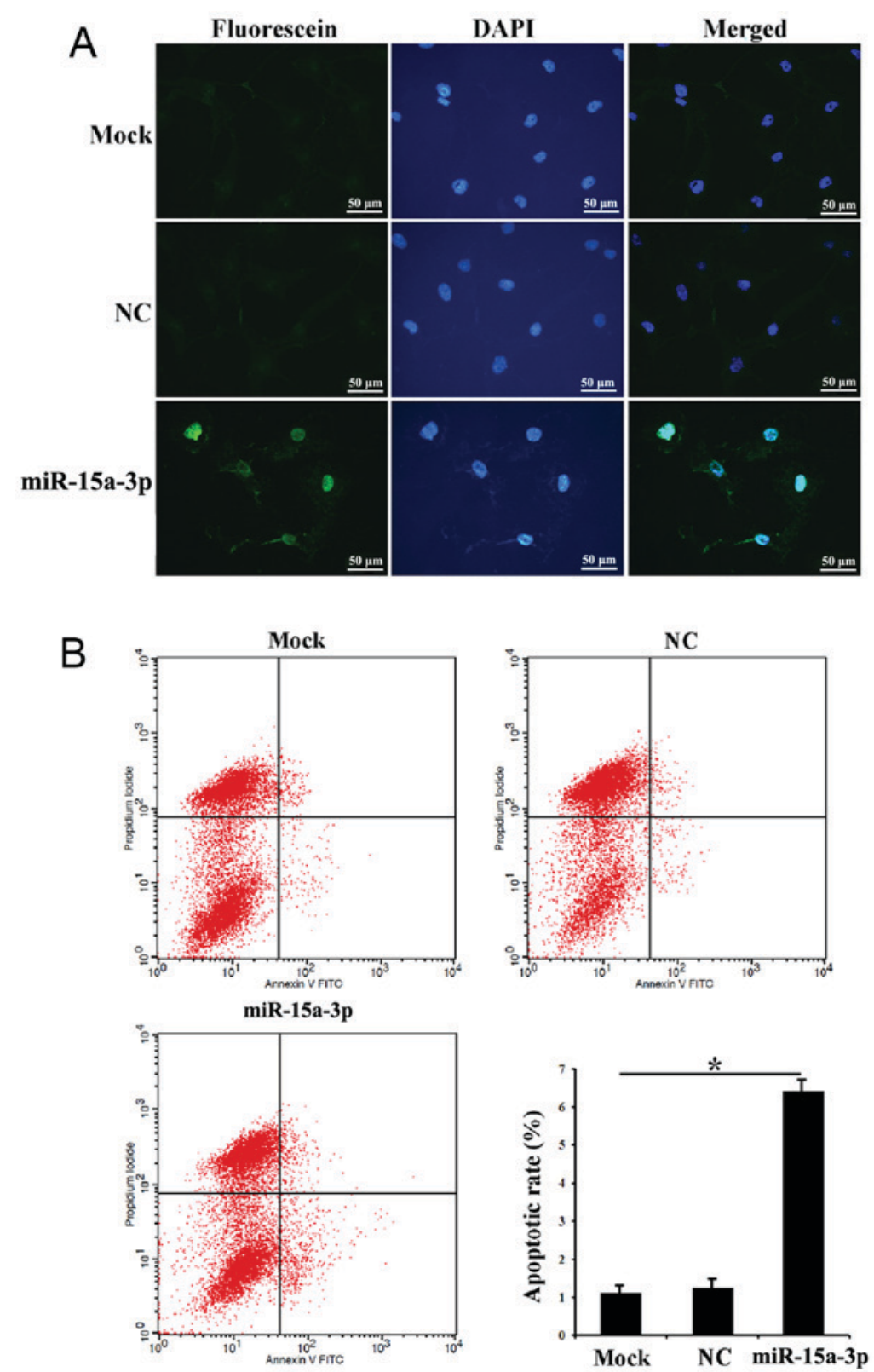

Figure 3. Expression of miR-15a-3p induces HLE-B3 cell apoptosis. (A) Terminal deoxynucleotidyl transferase dUTP nick end labeling assay of transfected HLE-B3 cells. (B) Cell apoptotic rates detected by flow cytometry. " $\mathrm{P}<0.01$. miR, microRNA; NC, negative control; DAPI, 4',6-diamidino-2-phenylindole. 

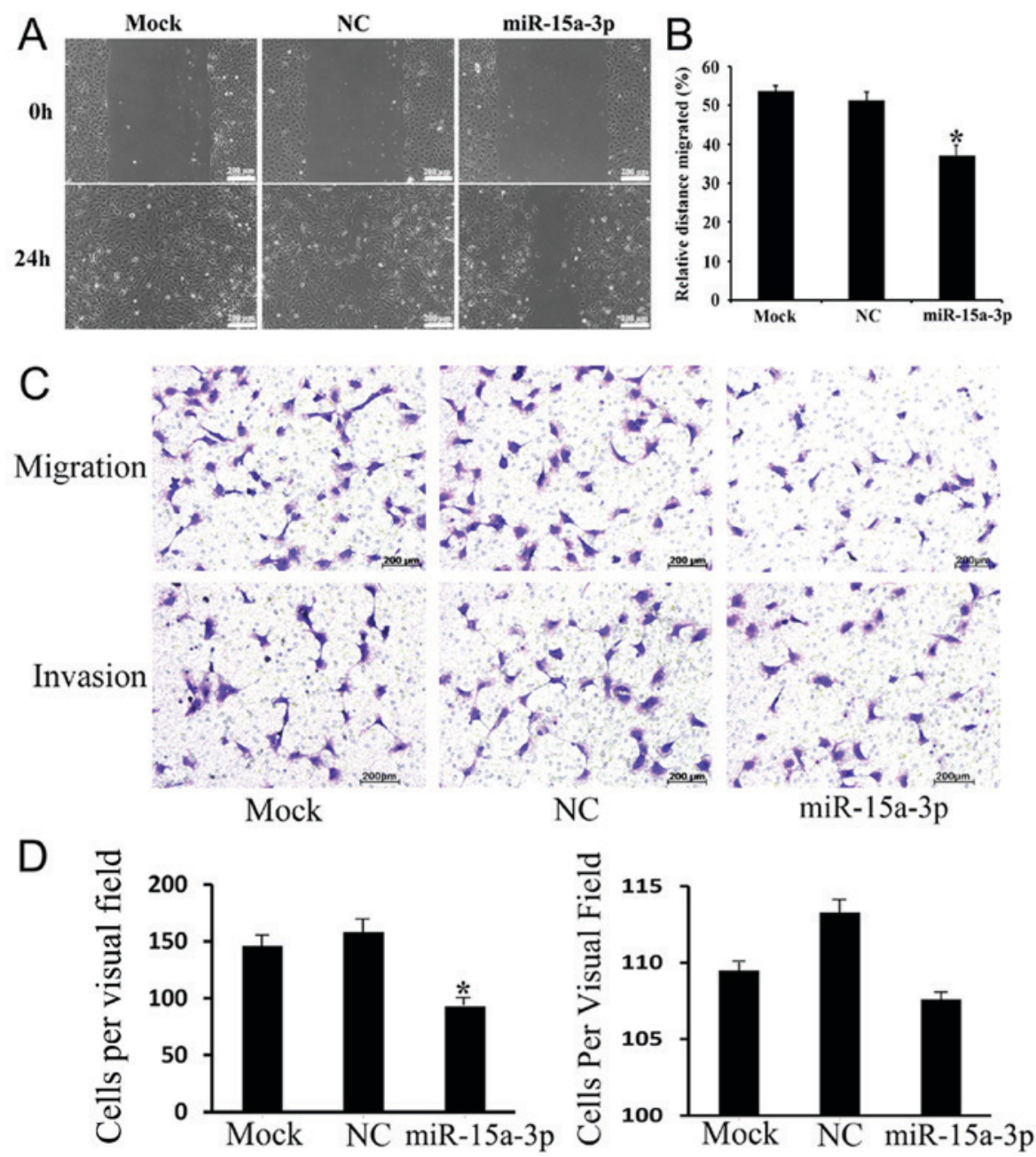

Figure 4. Expression of miR-15a-3p inhibits HLE-B3 cell migration. (A) Image and (B) relative migration distance comparison of the wound healing assay. (C) Image and (D) cell count of the Transwell assay showing cell migration and invasion. ${ }^{*} \mathrm{P}<0.01$. miR, microRNA; NC, negative control.
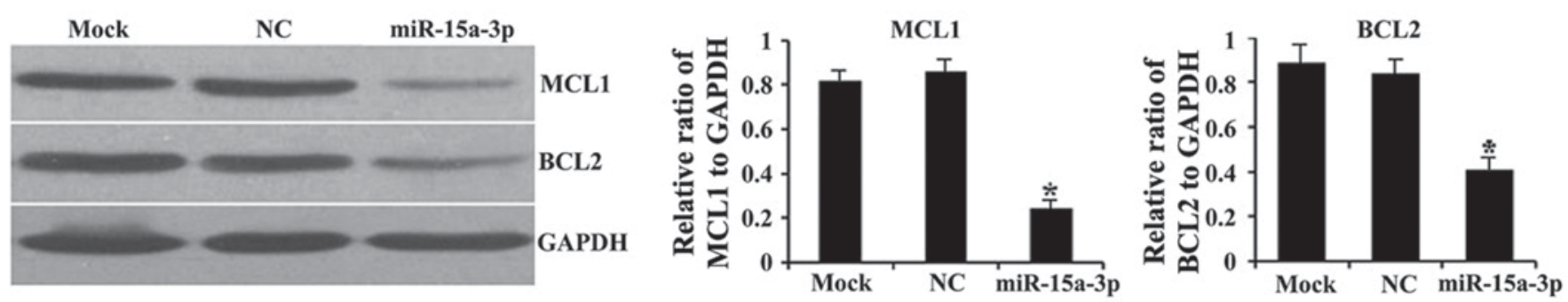

Figure 5. Relative protein expression of MCL1 and BCL2 justified against GAPDH. Results of the western blot analysis are shown. ${ }^{*}<<0.01$. miR, microRNA; NC, negative control; BCL2, B-cell lymphoma 2; MCL1, myeloid cell leukemia sequence 1.

double staining and flow cytometry, a significantly higher ratio of early apoptotic cells was found in the miR-15a cells than in the mock and NC cells. There was also more cell debris in the miR-15a cells than the NC cells (Fig. 3B).

Expression of miR-15a-3p inhibits HLE-B3 cell migration. To further investigate the effect of miR-15a-3p on HEL-B3 cell mobility in vitro, wound-healing and Transwell assays were performed. As the results of the wound-healing assay showed, the miR-15a cells were less migratory than the mock and NC cells (Fig. 4A and B). The Transwell migration and invasion assays revealed that the expression of miR-15a-3p inhibited the migration of HLE-B3 cells but did not affect their invasiveness to a significant level (Fig. 4C and D). These observations suggested that miR-15a-3p is important in reducing the migration of HLE-B3 cells in vitro.

miR-15a-3p downregulates the expression of BCL2 and $M C L 1$. To determine whether miR-15a-3p affects the intracellular expression of BCL2 and MCL1, western blot assays were performed on the transfected HLE-B3 cells. Significantly lower protein expression levels of BCL2 and MCL1 were observed in the miR-15a cells than in the mock and NC cells $(\mathrm{P}<0.01$; Fig. 5). 


\section{Discussion}

ARC is one of the major causes of eye disease leading to blindness $(10,11)$. The apoptosis of lens epithelial cells is the cellular basis for all types of cataracts, with the exception of congenital cataracts (2). However, the mechanism of lens epithelial cell apoptosis remains to be fully elucidated.

There have been reports suggesting that miRNAs are involved in the apoptosis of lens epithelial cells $(12,13)$. Peng et al identified the expression of miR-let-7b in lens epithelial cells from patients with ARC, and found that miRNA-let-7b was closely associated with the occurrence and development of ARC (14). Wu et al found no expression of miRNA-923 or miRNA-let-7b in normal human lens epithelial cells but significantly elevated expression of both in lens epithelial cells from patients with ARC (15). Previous studies have shown that miR-15a-3p is located at chromosome $13 q 14$ and that the deletion or downregulation of miR-15a-3p was associated with the origin and development of multiple types of cancer, including chronic lymphocytic lymphoma, prostate carcinoma and pituitary adenomas $(6,16,17)$. In addition, miR-15a-3p is known to act as a tumor suppressor, which can inhibit cell proliferation, promote the apoptosis of cancer cells, and suppress tumorigenesis in vitro and in vivo (18). However, the function of miR-15a-3p in ARC has not been reported.

Our preliminary RNA microarray identified apparently upregulated expression of miR-15a-3p in lens epithelial cells from patients with ARC (data not shown). In addition, 90 patients with clinical ARC were surveyed and found to have significantly upregulated expression levels of miR-15a-3p than normal individuals without ARC (7). Therefore, it was hypothesized that miR-15a-3p may be involved in the apoptosis of lens epithelial cells; however, its possible mechanism remained to be investigated.

miR-15a regulates the apoptosis and proliferation of cells by functioning in the regulation of multiple intracellular signaling pathways $(19,20)$. It was previously reported that miR-15a downregulated cyclin D1 and induced apoptosis and cell cycle arrest in osteosarcoma (21). In addition, the overexpression of miR-15a inhibited the growth and survival of multiple myeloma cells, suppressed cell migration and arrested cells at the G1 phase (22).

In normal lens epithelial cells, the expression of miR-15a is low; therefore, in the present study, lens epithelial cells were transfected with miR-15a-3p mimics in order to examine its biological function in lens epithelial cells. The results revealed increased apoptosis, and inhibition of proliferation and migration in HLE-B3 cells expressing miR-15a-3p mimics. The in vitro experiments suggested that miR-15a-3p may cause cataracts through promoting the apoptosis of lens epithelial cells.

The present study also examined the protein expression of two BCL2 family members. The BCL2 family is an important anti-apoptotic gene family, which mainly includes BCL2 BCL2-like 1, MCL1, BCL2-associated death promoter, and BCL2-associated X protein (23). This family can suppress cell apoptosis either through regulating cellular signal transduction by directly or indirectly affecting the release of $\mathrm{Ca}^{2+}$ in endoplasmic reticulum, or through suppressing lipid oxidation by oxygen free radicals (24). A previous study indicated that miR-15a was an important regulator of BCL2 (25). The 3'UTR of the BCL2 transcript has potential binding sites for miR-15a (25). Currently, investigations on BCL2 and MCL1 regulation by miR-15a are mainly focused on leukemia and malignant tumors $(4,26)$. Cimmino et al found that, in chronic lymphocytic leukemia, the expression of miR-15a was negatively correlated with BCL2, and that miR-15a negatively regulated BCL2 at the post-transcription level (4). In the present study, overexpressed miR-15a-3p mimics downregulated the expression level of BCL2 and MCL1. Our preliminary study also found a negative correlation between the level of miR-15a-3p and the expression of BCL2 and MCL1 in lens epithelial cells of patients with ARC (7). These results demonstrate that miR-15a-3p may induce the apoptosis of lens cells by downregulating the expression of BCL2 and MCL1.

One limitation of the present study was that the function and mechanism of miR-15a were only investigated in a single lens epithelial cell line. It remains unclear whether miRNA-15a has the same role in other lens epithelial cell lines. It would be beneficial to examine the involvement of miR-15a in the pathogenesis of cataracts using multiple lens cell lines in the future. In addition, the target gene of miR-15a was not verified, therefore, future investigations aim to confirm that BCL2 is a direct target of miR-15a. In addition, based on the results of our previous study (7), the role of hsa-miR-16-1-5p in human lens epithelial cells is currently under investigation.

In conclusion, the present study demonstrated that the overexpression of miR-15a-3p in vitro attenuated the proliferation of lens epithelial cells, and downregulated anti-apoptotic proteins BCL2 and MCL1, which may result in the apoptosis of lens epithelial cells. This study revealed a potential mechanism of miR-15a-3p regulating ARC and provide novel ideas for the effective prevention of cataracts.

\section{Acknowledgements}

Not applicable.

\section{Funding}

This study was funded by the Shandong Natural Science Foundation of China (grant no. ZR2015HL052), the Yantai Technology Development Plan (grant nos. 2017WS109, 2017YD005, 2013WS204, and 2010148-24), the Natural Science Foundation of China (grant no. 81701453) and the Youth Scientific Research Fund of Yantai Yuhuangding Hospital (grant no. 201502).

\section{Availability of data and materials}

The datasets used and/or analyzed during the present study are available from the corresponding author on reasonable request.

\section{Authors' contributions}

YBL and SJL were responsible for the concept and framework of the paper. SJL and WTW wrote the manuscript which was revised by YBL and NL. SJL and NL planned and performed the experiments. FLZ and YHY carried out RT-qPCR and 
western blotting. WTW, HJY and YL carried out cell proliferation, apoptosis, migration. All authors read and approved the final manuscript.

\section{Ethics approval and consent to participate}

The present study was approved by the Institutional Review Board of Yantai Yuhuangding Hospital.

\section{Patient consent for publication}

Not applicable.

\section{Competing interests}

The authors declare that they have no competing interests.

\section{References}

1. Ruotolo R, Grassi F, Percudani R, Rivetti C, Martorana D, Maraini G and Ottonello S: Gene expression profiling in human age-related nuclear cataract. Mol Vis 9: 538-548, 2003.

2. Li WC, Kuszak JR, Dunn K, Wang RR, Ma W, Wang GM, Spector A, Leib M, Cotliar AM and Weiss M: Lens epithelial cell apoptosis appears to be a common cellular basis for non-congenital cataract development in humans and animals. J Cell Biol 130: 169-181, 1995.

3. Ambros V: The functions of animal microRNAs. Nature 431: 350-355, 2004

4. Cimmino A, Calin GA, Fabbri M, Iorio MV, Ferracin M, Shimizu M, Wojcik SE, Aqeilan RI, Zupo S, Dono M, et al: miR-15 and miR-16 induce apoptosis by targeting BCL2. Proc Natl Acad Sci USA 102: 13944-13949, 2005.

5. Calin GA, Cimmino A, Fabbri M, Ferracin M, Wojcik SE Shimizu M, Taccioli C, Zanesi N, Garzon R, Aqeilan RI, et al: miR-15a and miR-16-1 cluster functions in human leukemia. Proc Natl Acad Sci USA 105: 5166-5171, 2008.

6. Aqeilan RI, Calin GA and Croce CM: miR-15a and miR-16-1 in cancer: Discovery, function and future perspectives. Cell Death Differ 17: 215-220, 2010.

7. Li Y, Liu S, Zhang F, Jiang P, Wu X and Liang Y: Expression of the microRNAs hsa-miR-15a and hsa-miR-16-1 in lens epithelial cells of patients with age-related cataract. Int J Clin Exp Med 8 : 2405-2410, 2015.

8. Livak KJ and Schmittgen TD: Analysis of relative gene expression data using real-time quantitative PCR and the 2(-Delta Delta C(T)) method. Methods 25: 402-408, 2001.

9. Lin C, Wang J, Wang Y, Zhu P, Liu X, Li N, Liu J, Yu L and Wang W: GRP78 Participates in PCA3-regulated prostate cancer progression. Anticancer Res 37: 4303-4310, 2017.

10. West S: Epidemiology of cataract: Accomplishments over 25 years and future directions. Ophthalmic Epidemiol 14: $173-178,2007$.
11. Foster A and Resnikoff S: The impact of Vision 2020 on global blindness. Eye (Lond) 19: 1133-1135, 2005.

12. Zhang F, Meng W and Tong B: Down-regulation of MicroRNA-133b suppresses apoptosis of lens epithelial cell by up-regulating BCL2L2 in age-related cataracts. Med Sci Monit 22: 4139-4145, 2016.

13. Dong Y, Zheng Y, Xiao J, Zhu C and Zhao M: MicroRNA let-7b induces lens epithelial cell apoptosis by targeting leucine-rich repeat containing $\mathrm{G}$ protein-coupled receptor 4 (Lgr4) in age-related cataract. Exp Eye Res 147: 98-104, 2016.

14. Peng CH, Liu JH, Woung LC, Lin TJ, Chiou SH, Tseng PC, Du WY, Cheng CK, Hu CC, Chien KH and Chen SJ: MicroRNAs and cataracts: Correlation among let-7 expression, age and the severity of lens opacity. Br J Ophthalmol 96: 747-751, 2012.

15. Wu C, Lin H, Wang Q, Chen W, Luo H, Chen W and Zhang H: Discrepant expression of microRNAs in transparent and cataractous human lenses. Invest Ophthalmol Vis Sci 53: 3906-3912, 2012.

16. Acunzo $M$ and Croce CM: Downregulation of miR-15a and miR-16-1 at 13q14 in Chronic Lymphocytic Leukemia. Clin Chem 62: 655-656, 2016.

17. Renjie W and Haiqian L: miR-132, miR-15a and miR-16 synergistically inhibit pituitary tumor cell proliferation, invasion and migration by targeting Sox 5. Cancer Lett 356: 568-578, 2015.

18. Huang E, Liu R and Chu Y: miRNA-15a/16: As tumor suppressors and more. Future Oncol 11: 2351-2363, 2015.

19. Lan F, Yue X, Ren G, Li H, Ping L, Wang Y and Xia T: miR-15a/16 enhances radiation sensitivity of non-small cell lung cancer cells by targeting the TLR1/NF- $\mathrm{KB}$ signaling pathway. Int J Radiat Oncol Biol Phys 91: 73-81, 2015.

20. Ye EA, Liu L, Jiang Y, Jan J, Gaddipati S, Suvas S and Steinle JJ: miR-15a/16 reduces retinal leukostasis through decreased pro-inflammatory signaling. J Neuroinflammation 13: 305, 2016.

21. Cai CK, Zhao GY, Tian LY, Liu L, Yan K, Ma YL, Ji ZW, Li XX, Han K, Gao J, et al: miR-15a and miR-16-1 downregulate CCND1 and induce apoptosis and cell cycle arrest in osteosarcoma. Oncol Rep 28: 1764-1770, 2012.

22. Hao M, Zhang L, An G, Sui W, Yu Z, Zou D, Xu Y, Chang H and Qiu L: Suppressing miRNA-15a/-16 expression by interleukin-6 enhances drug-resistance in myeloma cells. J Hematol Oncol 4: 37, 2011.

23. Vogler M, Walter HS and Dyer MJS: Targeting anti-apoptotic BCL2 family proteins in haematological malignancies-from pathogenesis to treatment. Br J Haematol 178: 364-379, 2017.

24. Frenzel A, Grespi F, Chmelewskij W and Villunger A: Bcl2 family proteins in carcinogenesis and the treatment of cancer. Apoptosis 14: 584-596, 2009.

25. Tang J, Wang Z, Chen L, Huang G and Hu X: Gossypol acetate induced apoptosis of pituitary tumor cells by targeting the BCL-2 via the upregulated microRNA miR-15a. Int J Clin Exp Med 8: 9079-9085, 2015

26. Xia L, Zhang D, Du R, Pan Y, Zhao L, Sun S, Hong L, Liu J and Fan D: miR-15b and miR-16 modulate multidrug resistance by targeting BCL2 in human gastric cancer cells. Int J Cancer 123: $372-379,2008$

(i) (5) This work is licensed under a Creative Commons Attribution-NonCommercial-NoDerivatives 4.0 International (CC BY-NC-ND 4.0) License. 